Research Paper

\title{
Expression of Histone Deacetylases in Diffuse Large B-cell Lymphoma and Its Clinical Significance
}

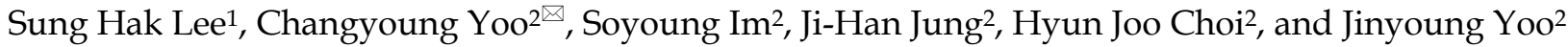 \\ 1. Department of Hospital Pathology, St. Mary's Hospital, The Catholic University of Korea, Seoul, Korea \\ 2. Department of Hospital Pathology, St. Vincent's Hospital, The Catholic University of Korea, Suwon, Korea
}

$\triangle$ Corresponding author: Changyoung Yoo, M.D., Ph.D., Department of Hospital Pathology, The Catholic University of Korea St. Vincent Hospital, 93, Jungbudaero, Paldal-gu, Suwon, Gyeonggi-do 442-723, Korea. Tel: 031-249-7646; Fax: 031-244-6786; E-mail: ppgg2@catholic.ac.kr

( ) Ivyspring International Publisher. This is an open-access article distributed under the terms of the Creative Commons License (http://creativecommons.org/ licenses/by-nc-nd/3.0/). Reproduction is permitted for personal, noncommercial use, provided that the article is in whole, unmodified, and properly cited.

Received: 2014.01.07; Accepted: 2014.07.01; Published: 2014.07.17

\begin{abstract}
Background: Histone deacetylase inhibitors are a new class of drugs used in treatment of malignant tumors. Diffuse large B-cell lymphoma (DLBCL) is the most common type of B-cell lymphoma, and it accounts for more than $40 \%$ of all B-cell lymphomas. In this study, we aimed to determine the expression patterns of histone deacetylases (HDACs) in DLBCL, to examine whether HDAC expression patterns differ among cases, and to assess whether these findings have clinical significance.

Materials and methods: We selected 91 cases of DLBCL diagnosed at St. Vincent Hospital, The Catholic University of Korea, from 200I-20I2. We performed a pathology slide review and collected clinical data including age, sex, tumor site, survival time, and mortality. Immunohistochemical analysis was performed using primary antibodies for HDACs, including $\mathrm{HDACl}$ and 2 of class I, HDAC4 and 5 of class Ila, and HDAC 6 of class llb. Expression site was determined to be nuclear, cytoplasmic, or both. Staining intensities were graded as low and high. We assessed correlations between HDAC expression levels and clinical data and survival analysis.

Results: Of the 91 cases examined, 46 (50.5\%) were men and 45 (49.5\%) were women. Most of the patients were elderly, and $74(81.3 \%)$ cases were older than 46 y. Forty-six $(50.5 \%)$ cases showed lymph node involvement, and 45 (49.5\%) cases showed lymphoma at extranodal sites. In nodal lymphoma, staining was strongly positive for HDAC2, whereas staining was weak or negative for HDAC4; however, there was no significant correlation with survival. But nodal lymphoma cases with high nuclear expression of HDAC2 and nodal lymphoma cases with high nuclear expression of HDAC2 and low nuclear expression of HDAC4 showed significantly shorter survival times compared with other cases.

Conclusions: High nuclear expression of HDAC2 may play an important role in survival of DLBCL patients, especially in those with nodal lymphoma, which is associated with a shorter survival time. Our results may have important implications for treatment of DLBCL by epigenetic regulation.
\end{abstract}

Key words: Diffuse large B-cell lymphoma; Histone deacetylase; Epigenetic; Nodal lymphoma; Survival

\section{Introduction}

Epigenetic dysregulation affects gene expression, including methylation, histone modification, and small silent RNAs (siRNAs) [1, 2]. These epige- netic alterations can serve as targets for various drugs, which can normalize gene transcription [1]. Histones are most commonly modified by acetylation, which 
determines the chromatin structure and influences gene transcription. Acetylation is known to stimulate gene transcription, and deacetylation is known to inhibit gene transcription because it produces more a compact chromatin structure [3]. Acetylation of histones is regulated by the antagonistic actions of two groups of enzymes, known as histone acetyltransferases (HATs) and histone deacetylases (HDACs); it is well known that HDACs have highly specific functions in gene regulation [3]. The HDAC superfamily is known to be composed of 11 proteins, which can be classified as follows: class I HDACs are ubiquitous and include HDAC1, 2, 3, and 8; class IIa includes HDAC4, 5, 7, and 9; and class IIb includes HDAC6 and 10. $[2,3,4-6]$. Additional deacetylases, including SIRTUINS 1-7, are sometimes grouped as class III, but they are dealt differently with HDACs [2].

Histone deacetylase inhibitors are newly introduced agents for the treatment of hematologic malignancies, and their principal mechanisms of action involve functional modification of neoplastic cells via their effects on the acetylation of histones and non-histone proteins [7-10]. Their precise mechanisms of action are not fully understood, but several hypotheses have been proposed, and generally, these hypotheses suggest blocking of gene expression and inhibition of the growth in neoplastic cells $[3,7]$. These inhibitors act on histone and non-histone proteins and regulate gene expression and cellular functions through various pathways $[2,3]$. These inhibitors are particularly involved in regulation of the cell cycle, inhibition of cell growth, apoptosis, and angiogenesis $[1,2,5,11]$. For example, romidepsin, a histone deacetylase inhibitor, causes arrest of the cell cycle between the G1 and G2/M phases, stimulation of cellular differentiation, increased expression of $\mathrm{p} 21$ and cycle E, decreased expression of cyclin D and c-myc, stimulation of apoptosis, and inhibition of phosphorylation of the $\mathrm{Rb}$ gene [1]. Collectively, HDACs are closely and primarily related to factors involved in the regulation of the cell cycle and apoptosis such as p53, various cyclins, and Bcl-6 [11-13]. Therefore, we suggest that HDAC inhibitors have an indirect effect on these factors. Although HDACs typically inhibit transcription, in certain situations they activate transcription and act on non-histone proteins located in the cellular matrix. In addition, many other factors are involved in the acetylation and deacetylation process. How these factors regulate gene expression is an important question [3].

Because various histone deacetylase inhibitors are a novel and effective class of drugs for the treatment of malignant tumors, determining the expression patterns of HDACs in malignant lymphomas may facilitate development of more effective treat- ments. B-cell lymphomas are the most common type of lymphoma, and diffuse large B-cell lymphoma (DLBCL) is the most common type of B-cell lymphoma accounting for more than $40 \%$ of all B-cell lymphomas [14]. DLBCL is an aggressive form of non-Hodgkin lymphoma, and it commonly arises in the lymph nodes; however, extranodal DLBCL is also frequent. In addition, there are various histologic subtypes of DLBCL, and their subclassification is becoming increasingly complex [14]. This study aimed to determine the expression patterns of HDACs in DLBCL, to assess whether HDAC expression patterns differ among cases, and to examine whether these findings have clinical significance.

\section{Materials and methods}

For this study, we selected 91 cases of DLBCL diagnosed from 2001-2012 at St. Vincent's Hospital, The Catholic University of Korea, which had well-preserved paraffin blocks and in which auxiliary immunohistochemical studies were performed to establish the diagnosis of DLBCL. We performed a pathology slide review of both the HE and immunohistochemistry slides. Clinical data including sex, age, tumor site, survival time, and mortality were collected from the clinical records. For analysis, we arbitrarily classified patient age as younger or older than $45 \mathrm{y}$, and tumor site as nodal or extranodal lymphoma. Immunohistochemical analysis was performed using primary antibodies for the HDACs, including HDAC1 (monoclonal; 1:800; SantaCruz, TX, USA) and HDAC2 (monoclonal; 1:400; SantaCruz, TX, USA) of class I, HDAC4 (monoclonal; 1:100; SantaCruz, TX, USA) and HDAC5 (monoclonal; 1:400; SantaCruz, TX, USA) of class IIa, and HDAC6 (monoclonal; 1:25; SantaCruz, TX, USA) of class IIb. Using control tissue such as lymph nodes, we determined the optimal dilution of these antibodies. The procedures used for the immunohistochemical analysis were as follows: Using a formalin-fixed paraffin-embedded tissue block, we used a 2.0-mm sample from each to construct a tissue microarray (TMA). After deparaffinization of TMA slides using heat and xylene, samples were rehydrated by serial ethanol soaking. Antigen retrieval was performed by boiling in retrieval solution, and inactivation of endogenous peroxidase using 3\% hydrogen peroxide was performed in sequence. The primary antibodies mentioned above were applied and a secondary antibody reaction was performed. EnVision (Dako, Glostrup, Denmark) and DAB were used as the secondary antibody system and the chromogen, respectively. After counterstaining with Mayer's hematoxylin, slides were mounted.

We determined whether the various HDACs were expressed in the nucleus or the cytoplasm. As 
described in the Results below, the expression of the HDACs used in this study showed an all or nothing pattern, i.e. the degree of expression was homogenous and could not be estimated using percentages. Therefore, we divided the degree of expressions into 4 grades based on staining intensity: Grade 0 when none of the cells were stained, Grade 1 when only a few cells were stained, Grade 2 when most of the cells were stained but the intensity was weak, and Grade 3 when most of the cells were stained with strong intensity. This grading system was applied to both the nuclear and cytoplasmic staining. We classified Grades 0 and 1 as low staining intensity, and Grades 2 and 3 as high (Fig. 1, 2). After analysis, correlations with clinical data such as sex, age, tumor site, and survival analysis were determined. We used the chi-square test and Kaplan-Meier survival analysis to analyze the statistical significance of the results, using SAS software (version 8; SAS Inc., Cary, NC, USA). A $p$-value of $<0.05$ was considered significant. This study was approved by the Clinical Study Medical Ethics Committee (VC11SISI0087).

\section{Results}

Of the 91 cases, 46 (50.5\%) were men and 45 $(49.5 \%)$ were women. Most of the patients (74 cases, $81.3 \%)$ were older than $46 \mathrm{y}$. Seventeen $(18.7 \%)$ of the cases were younger than 45 y. Forty-six $(50.5 \%)$ cases showed lymph node involvement and 45 (49.5\%) cases showed lymphoma at extranodal sites. The most common extranodal sites were the small intestine, stomach, soft tissue, and testis (Table 1). In general, DLBCL was more common in the elderly, and showed similar proportions in men and women. Proportions of nodal and extranodal DLBCL were also similar.

HDAC1 was faintly expressed in the nucleus in normal lymph node tissue used as a control. In the cases studied, HDAC1 was expressed in nucleus in only $12(13.2 \%)$ cases with faint staining intensity. HDAC1 staining was not observed in the cytoplasm (Fig. 1, A-C). HDAC2 was expressed exclusively in the nucleus both in control tissue and in the cases studied. High nuclear expression was found in 62 $(68.1 \%)$ cases, and the remaining $29(31.9 \%)$ cases showed low nuclear expression. HDAC2 staining was not observed in the cytoplasm (Fig. 1, D-H). HDAC4, 5 , and 6 were expressed in both the nucleus and cytoplasm at varying intensities not only in control tissues but also in the cases studied. HDAC4 showed high expression in the nucleus and the cytoplasm in $34(37.4 \%)$ and $56(61.5 \%)$ cases, respectively, and low expression in the nucleus and the cytoplasm in 57 $(62.6 \%)$ and $35(38.5 \%)$ cases, respectively (Fig. 2, A-E). HDAC5 showed high expression in the nucleus and the cytoplasm in $73(80.2 \%)$ and $53(58.2 \%)$ cases, respectively, and low expression in the nucleus and the cytoplasm in $18(19.8 \%)$ and $38(41.8 \%)$ cases, respectively (Fig. 2, F-J). HDAC6 showed high expression in the nucleus and the cytoplasm in $37(40.7 \%)$ and $81(89.0 \%)$ cases, respectively, and low expression in the nucleus and the cytoplasm in $54(59.3 \%)$ and 10 $(11.0 \%)$ cases, respectively (Fig. 2, K-O). In general, HDAC4 showed similar patterns of expression pattern in the nucleus and cytoplasm, but HDAC5 and HDAC6 were preferentially expressed in the nucleus and cytoplasm, respectively (Table 2).

Table I. Clinical characteristics of the cases studied

\begin{tabular}{|c|c|c|c|}
\hline & & Proportion (\%) & Total \\
\hline \multirow[t]{3}{*}{ Sex } & $\mathrm{M}$ & 46 (50.5) & \\
\hline & $\mathrm{F}$ & 45 (49.5) & \\
\hline & & & 91 \\
\hline \multirow[t]{3}{*}{ Age } & $\leq 45$ & 17 (18.7) & \\
\hline & $\geq 46$ & $74(81.3)$ & \\
\hline & & & 91 \\
\hline \multirow[t]{21}{*}{ Site } & Nodal & $46(50.5)$ & \\
\hline & Extranodal & 45 (49.5) & \\
\hline & Bone & 1 & \\
\hline & Brain & 2 & \\
\hline & Breast & 2 & \\
\hline & Colon & 2 & \\
\hline & Kidney & 1 & \\
\hline & Mediastinum & 1 & \\
\hline & Nasal cavity & 2 & \\
\hline & Ovary & 2 & \\
\hline & Parotid gland & 1 & \\
\hline & Small intestine & 8 & \\
\hline & Soft tissue & 5 & \\
\hline & Stomach & 5 & \\
\hline & Testis & 4 & \\
\hline & Thyroid gland & 1 & \\
\hline & Tongue & 1 & \\
\hline & Tonsil & 4 & \\
\hline & Urinary bladder & 2 & \\
\hline & Uterus & 1 & \\
\hline & & & 91 \\
\hline
\end{tabular}

Table 2. Expression patterns of HDACs

\begin{tabular}{|c|c|c|c|c|}
\hline \multicolumn{4}{|c|}{ Frequency (\%) } & \multirow[b]{2}{*}{ Total } \\
\hline HDAC & Expression site & Low & High & \\
\hline \multirow[t]{2}{*}{1} & Nucleus & $12(13.2)$ & 0 & $12(13.2)$ \\
\hline & Cytoplasm & 0 & 0 & 0 \\
\hline \multirow[t]{2}{*}{2} & Nucleus & 29 (31.9) & $62(68.1)$ & $91(100)$ \\
\hline & Cytoplasm & 0 & 0 & 0 \\
\hline \multirow[t]{2}{*}{4} & Nucleus & $57(62.6)$ & $34(37.4)$ & $91(100)$ \\
\hline & Cytoplasm & $35(38.5)$ & $56(61.5)$ & $91(100)$ \\
\hline \multirow[t]{2}{*}{5} & Nucleus & 18 (19.8) & $73(80.2)$ & $91(100)$ \\
\hline & Cytoplasm & 38 (41.8) & $53(58.2)$ & $91(100)$ \\
\hline \multirow[t]{2}{*}{6} & Nucleus & $54(59.3)$ & 37 (40.7) & $91(100)$ \\
\hline & Cytoplasm & $10(11.0)$ & $81(89.0)$ & 91 (100) \\
\hline
\end{tabular}




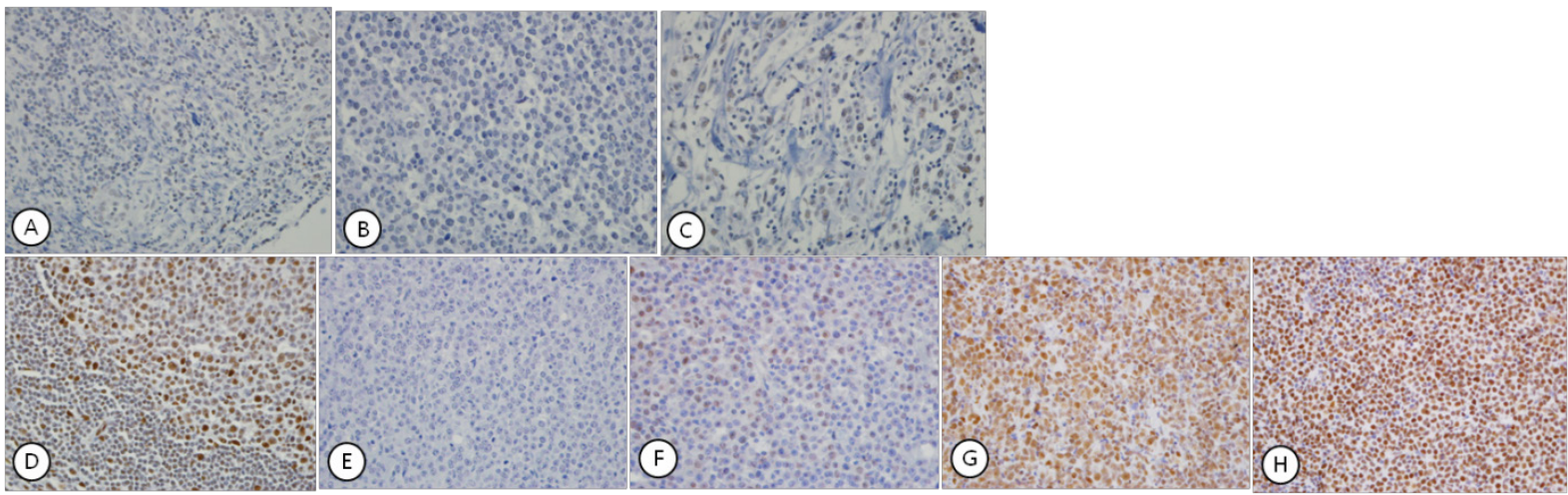

Fig. I. Expression patterns of HDACI $(A-C)$ and HDAC2 (D-H), which showed exclusively nuclear staining. $\mathrm{HDACl}$ showed faint staining in the nucleus of normal lymphocytes in control tissue (A) and a few lymphoma cells (B). Most tumor cells were negative for HDACl (C). HDAC2 showed strong nuclear staining in lymphocytes of control tissue (D). Nuclear staining in the lymphoma cells was graded as Grade $0(E)$, Grade I (F), Grade $2(G)$, and Grade $3(H)$ according to the intensity of nuclear staining.

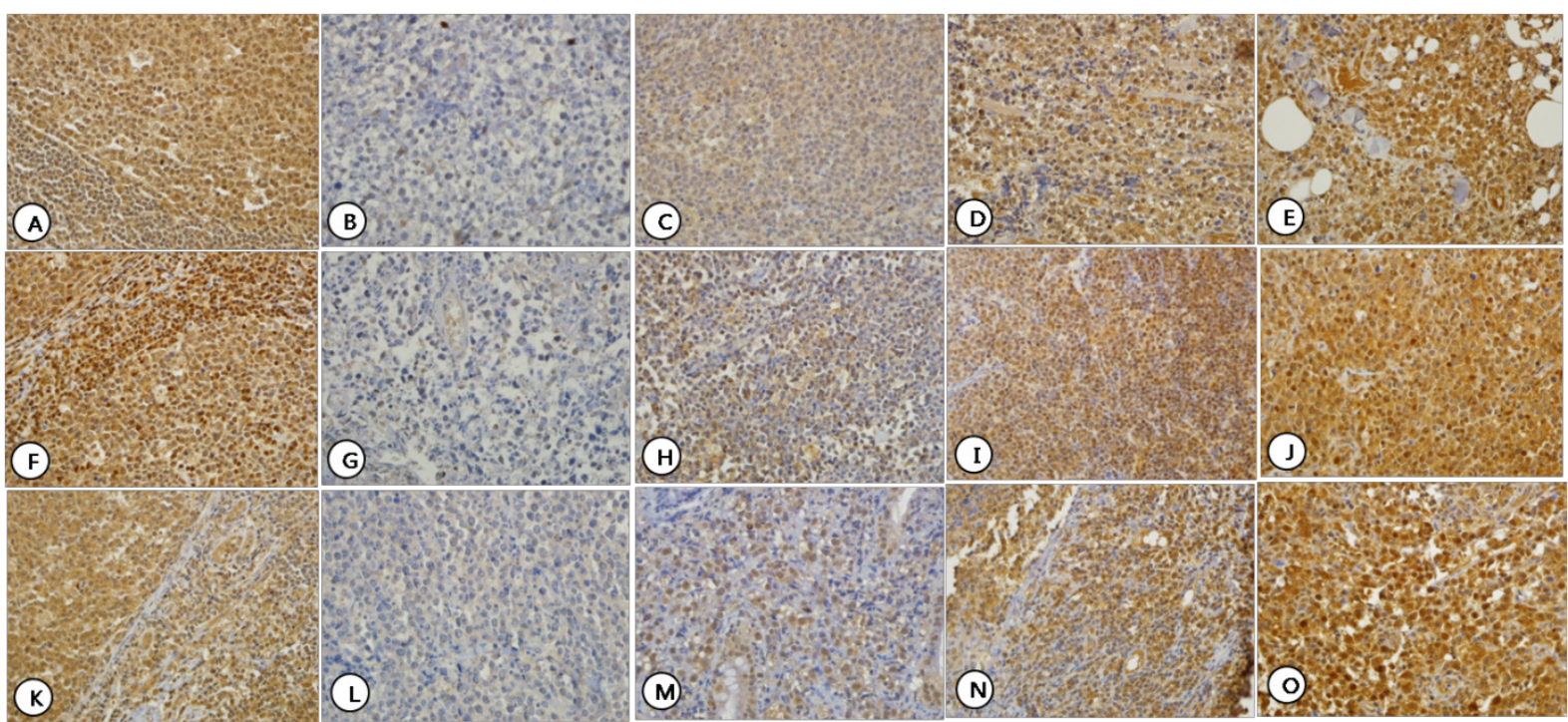

Fig. 2. Expression patterns of HDAC4 (A-E), HDAC5 (F-J), and HDAC6 (K-O) which showed both nuclear and cytoplasmic staining. Normal lymphocytes in control tissue showed nuclear and cytoplasmic staining for HDAC4 (A), HDAC5 (F) and HDAC6 (K). For each HDAC, staining intensity was graded as Grade 0-3. Grade 0: HDAC4 (B), HDAC5 (G), and HDAC6 (L); Grade I: HDAC4 (C), HDAC5 (H), and HDAC6 (M); Grade 2: HDAC4 (D), HDAC5 (I), and HDAC6 (N); and Grade 3: HDAC4 (E), HDAC5 (J), and HDAC6 (O).

Analysis of correlations with clinical characteristics showed that HDAC2 was expressed similarly in men and women. High cytoplasmic expression of HDAC4 $(p=0.022)$ and high nuclear $(p=0.016)$ and cytoplasmic $(p=0.042)$ expression of HDAC5 were more frequently observed in women, but the clinical significance is uncertain. HDAC expression did not show a significant correlation with age. Expression patterns differed slightly between nodal and extranodal lymphomas. In nodal lymphomas, there was a strong nuclear positivity for HDAC2 $(p=0.011)$, whereas there was negativity or a weak nuclear positivity for HDAC4 $(p=0.000)$. Significant differences were not observed between HDAC1, 5, and 6 (Table 3). HDAC expression may differ somewhat between nodal and extranodal DLBCL.

Survival analysis showed no differences with respect to age or sex. Survival duration differed be- tween nodal and extranodal DLBCL, at $55.531 \pm 6.906$ and $72.134 \pm 6.359$ months, respectively, but the result was not statistically significant $(p=0.132)$. When survival analysis was made with respect to the expression patterns of HDACs, cases of nodal lymphomas with a high nuclear expression of HDAC2 and a low nuclear expression of HDAC4, which showed a statistical significance in relation to the expression patterns, were not significantly correlated with the results of the survival analysis, although these cases may show a marginally shorter survival period (Table 4 and Fig. 3). Nor were expression patterns of HDAC5 and 6 significantly correlated with survival times. However, analysis of correlations between tumor site and HDAC expression patterns showed that in nodal lymphoma cases with high nuclear expression of HDAC2 and in nodal lymphoma cases with both high nuclear HDAC2 and low nuclear HDAC4 expression 
survival time was significantly shorter than in other cases ( $p=0.018$ and 0.006 , respectively). Nodal lymphoma cases with low nuclear HDAC4 expression showed a tendency toward a shorter survival time, but this result was not statistically significant $(p=$ 0.092) (Table 4 and Fig. 4).

Table 3. Correlations between HDAC expression and clinical characteristics

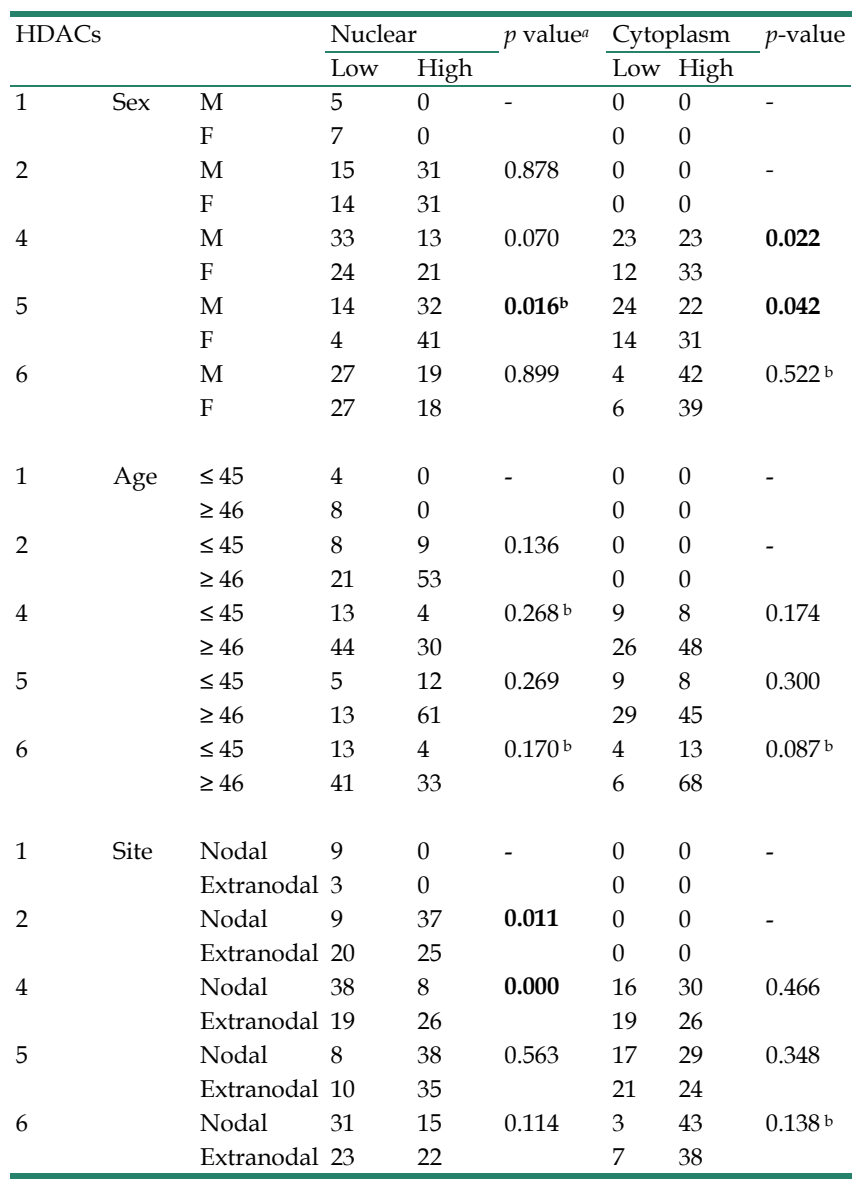

a. Pearson's chi-square test

b. Fisher's exact test

\section{Discussion}

In this study we concluded that high nuclear expression of HDAC2 may play an important role in survival of DLBCL patients, especially in those with nodal lymphoma, which is associated with a shorter survival time. Our results may have important implications for treatment of DLBCL by epigenetic regulation. It is well known that HDACs, which are the primary subject of this study, have highly specific functions in gene regulation [3].

Several studies reported on the clinical applications of HDAC are in treatment of lymphomas. HDAC6 is the HDAC most commonly expressed in various lymphomas [2]. Available treatments for lymphomas include CHOP, monoclonal antibodies, radiotherapy, immune regulators, and proteasome inhibitors; in addition, several new experimental agents have been introduced and applied in clinical practice. Among them, histone deacetylase inhibitors are considered to be promising agents $[2,5,6,8]$. At present, several inhibitors, including SAHA and romidepsin, are used for treatment of cutaneous T-cell lymphoma [1, 3, 11]. In addition, several trials for B-cell lymphomas have been reported, but these treatments are in the early stages of development compared with those for T-cell lymphomas $[2,15]$. In this study, we attempted to determine the expression patterns of HDACs in DLBCL, the most common and probably the most divergent lymphoma, and to examine whether these findings have clinical significance. For the selection of HDACs we referred to previous reports that dealt with the relationship between HDACs and lymphomas. These studies most frequently examined HDAC1, 2, 3, and 6 [16-18]. In this study, we selected well-studied HDACs including HDAC1, 2, and 6. Because the role of class IIa HDACs in lymphoma has not been studied sufficiently, we also included HDAC4 and 5 . To gain a more complete understanding of the contributions made by HDACs in DLBCL, it will be necessary to perform additional studies examining other types of HDACs.

Table 4. Correlations between survival analysis and clinical parameters and expression of various HDACs

\begin{tabular}{|c|c|c|c|c|}
\hline \multirow{2}{*}{\multicolumn{2}{|c|}{ Sex }} & \multicolumn{2}{|c|}{ Mean survival $(\mathrm{mo}) \pm \mathrm{SD}$} & \multirow[t]{2}{*}{ p-value } \\
\hline & & $\mathrm{M}$ & $\mathrm{F}$ & \\
\hline & & $67.754 \pm 6.707$ & $63.987 \pm 6.867$ & 0.941 \\
\hline \multirow{2}{*}{\multicolumn{2}{|c|}{ Age }} & $\leq 45$ & $\geq 46$ & \\
\hline & & $70.203 \pm 9.970$ & $62.865 \pm 5.602$ & 0.328 \\
\hline \multirow{2}{*}{\multicolumn{2}{|c|}{ Sites }} & Nodal & Extranodal & \\
\hline & & $55.531 \pm 6.906$ & $72.134 \pm 6.359$ & 0.132 \\
\hline \multicolumn{2}{|l|}{ HDACs } & Low & High & \\
\hline 2 & Nucleus & $75.269 \pm 7.571$ & $58.715 \pm 5.793$ & 0.232 \\
\hline \multirow[t]{2}{*}{4} & Nucleus & $61.845 \pm 6.511$ & $71.074 \pm 6.521$ & 0.225 \\
\hline & Cytoplasm & $62.171 \pm 8.017$ & $67.174 \pm 5.579$ & 0.382 \\
\hline \multirow[t]{2}{*}{5} & Nucleus & $65.602 \pm 10.827$ & $64.001 \pm 5.069$ & 0.765 \\
\hline & Cytoplasm & $62.437 \pm 7.151$ & $66.675 \pm 6.526$ & 0.800 \\
\hline \multirow[t]{2}{*}{6} & Nucleus & $63.646 \pm 5.806$ & $64.246 \pm 8.166$ & 0.834 \\
\hline & Cytoplasm & $68.488 \pm 11.110$ & $66.641 \pm 5.536$ & 0.909 \\
\hline \multirow{6}{*}{\multicolumn{2}{|c|}{ Sites and HDACs }} & Nodal and $2 \mathrm{NH}^{\mathrm{b}}$ & Others & \\
\hline & & $50.357 \pm 7.652$ & $74.564 \pm 5.812$ & 0.018 \\
\hline & & Nodal and $4 \mathrm{NLC}^{\mathrm{c}}$ & Others & \\
\hline & & $50.316 \pm 7.377$ & $72.230 \pm 5.733$ & 0.092 \\
\hline & & $\begin{array}{l}\text { Nodal and } 2 \mathrm{NH} \\
\& 4 \mathrm{NL}^{\mathrm{d}}\end{array}$ & Others & \\
\hline & & $43.233 \pm 8.145$ & $74.263 \pm 5.307$ & 0.006 \\
\hline
\end{tabular}

a. Log Rank

b. Nodal lymphoma cases showing high nuclear expression of HDAC2. c. Nodal lymphoma cases showing low nuclear expression of HDAC4.

d. Nodal lymphoma cases showing high nuclear expression of HDAC2 but low nuclear expression of HDAC4. 

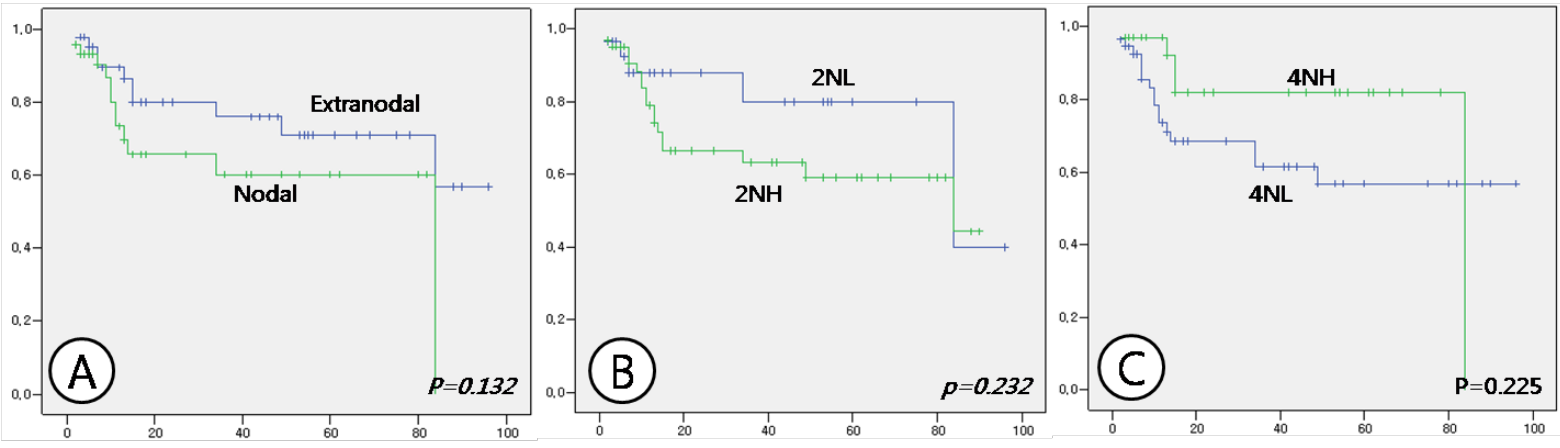

Duration (months)

Fig. 3. Comparison of survival between nodal and extranodal lymphoma cases $(A)$, between nuclear expression of HDAC2 at low (2NL) and high (2NH) intensity (B), and between nuclear expression of HDAC4 at high (4NH) and low (4NL) intensity (C).
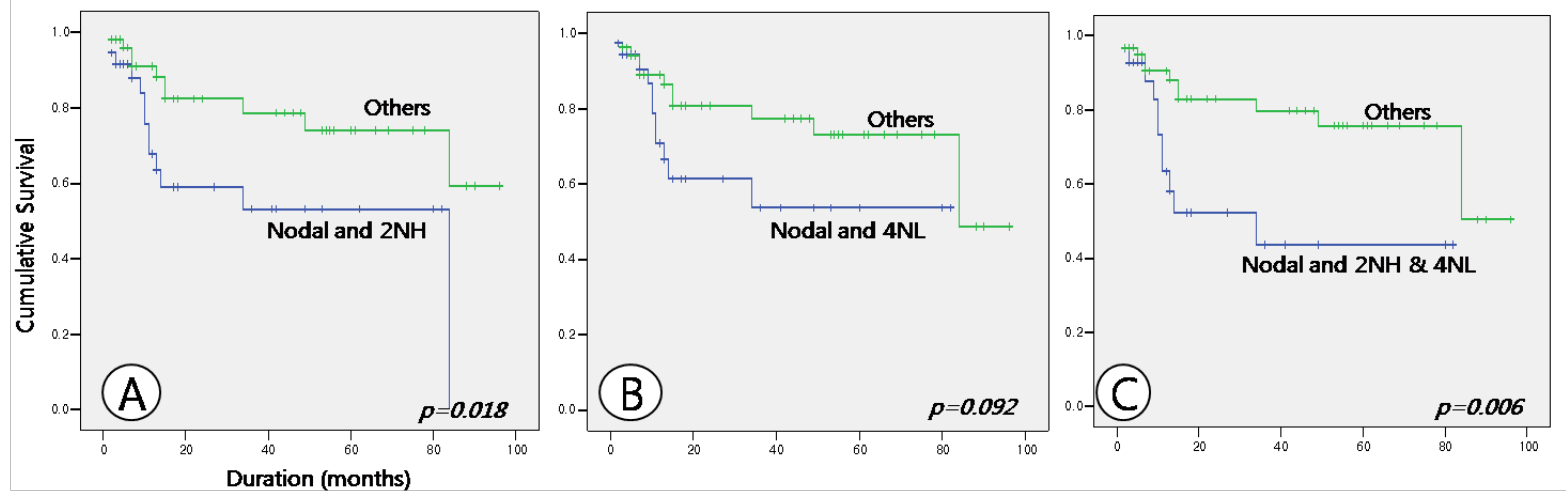

Fig. 4. Comparison of survival between nodal lymphoma cases with high nuclear expression of HDAC2 (Nodal and $2 \mathrm{NH})$ and others (A), between nodal lymphoma cases with low nuclear expression of HDAC4 (Nodal and 4NL) and others (B), and between nodal lymphoma cases with both high nuclear expression of HDAC2 and low nuclear expression of HDAC4 (Nodal and 2NH \& 4NL) and others (C).

The sex ratio in cases of DLBCL in this study was nearly equal. We observed that DLBCL was more frequent in elderly patients, as is already well known [14]. DLBCL almost equally involved the lymph nodes and extranodal sites. HDACs of class I, especially HDAC2, appear to be important in DLBCL. In this study, high nuclear expression of HDAC2 was more frequent in nodal lymphomas, and nodal lymphomas with high nuclear expression of HDAC2 were associated with a shorter survival time. Although it was not statistically significant, nodal lymphomas showed a tendency towards a poorer prognosis than extranodal lymphomas; however, when HDAC2 expression was assessed, the clinical significance became clear. Thus, it appears that there are different epigenetic mechanisms especially for the expression of HDACs between nodal and extranodal lymphomas. We supposed that class I HDACs are restrictively expressed in DLBCL, but high nuclear expression of class I HDACs is associated with a shorter survival time. HDACs of other classes appeared to be expressed more frequently than class I HDACs, but their clinical significance appeared to be limited. In this study, low nuclear expression of HDAC4 was more frequent in nodal lymphomas, but nodal lym- phoma cases with low nuclear HDAC4 expression did not show a statistically significant difference in survival, although a tendency towards shorter survival time was observed. Other HDACs of class IIa and IIb were insignificant not only in their expression patterns but also in functions relevant for survival. Comparison of nodal lymphomas with both high nuclear expression of HDAC2 and low nuclear expression of HDAC4 with other cases showed that survival time of the former cases was significantly shorter. However, it is unclear whether this result was obtained because of HDAC2 or HDAC4. It may reasonable to conclude that HDAC2 of class I plays an important role in DLBCL with respect to survival, and that HDACs of other classes play accessory roles. The role of HDAC2 in malignant tumors has not been sufficiently studied. HDAC2 is known to be associated with hematologic malignancies such as classical Hodgkin's lymphoma, cutaneous T-cell lymphoma, and DLBCL $[16,18,19]$. Associations with non-hematologic malignancies such as breast cancer, hepatocellular carcinoma, and gallbladder cancer have also been reported [20-22].

Collectively, high nuclear expression of HDAC2 was more frequent in nodal lymphomas than in ex- 
tranodal lymphomas, and nodal lymphoma with high nuclear expression of HDAC2 was associated with shorter survival than in other cases. The importance of other HDACs in survival was unclear, with the exception of HDAC4. Currently, histone deacetylase inhibitors are used for treating certain types of T-cell lymphomas, but on the basis of our study, these inhibitors may also be useful for the treatment of DLBCL, especially the nodal type. Some studies have reported on the role of HDAC2 in DLBCL, but to the best of our knowledge, this is the first study to report prognostic differences on the basis of the level and site (nodal or extranodal) of HDAC2 expression. Therefore, although HDAC2 has been well studied in the field of lymphoma, the results of this study provide novel, basic information important to future study of DLBCL, the most important and divergent type of lymphoma. These findings reflect the diversity of DLBCL. As in T-cell lymphoma, these HDACs and their inhibitors may also play a role in cell cycle regulation and apoptosis [23]. HDACs have also been suggested to be closely related to MHC class II expression [24]. Further study of the relationship between HDACs and factors involved in cell cycle regulation and apoptosis in DLBCL is required for development of effective treatments.

\section{Competing Interests}

The authors have declared that no competing interest exists.

\section{References}

1. Jain N, Odenike O. Emerging role of the histone deacetylase inhibitor romidepsin in hematologic malignancies. Expert Opin Pharmacother. 2010; 11: 3073-84.

2. Zain J, O'Connor OA. Targeting histone deacetyalses in the treatment of Band T-cell malignancies. Invest New Drugs. 2010; 28 Suppl 1: S58-78.

3. Haberland M, Montgomery RL, Olson EN. The many roles of histone deacetylases in development and physiology: implications for disease and therapy. Nat Rev Genet. 2009; 10: 32-42.

4. Watanabe T. Investigational histone deacetylase inhibitors for non-Hodgkin lymphomas. Expert Opin Investig Drugs. 2010; 19: 1113-27.

5. Buglio D, Younes A. Histone deacetylase inhibitors in Hodgkin lymphoma. Invest New Drugs. 2010; 28 Suppl 1: S21-7.

6. Copeland A, Buglio D, Younes A. Histone deacetylase inhibitors in lymphoma. Curr Opin Oncol. 2010; 22: 431-6.

7. Mithraprabhu S, Grigoriadis G, Khong T, et al. Deacetylase inhibition in myeloproliferative neoplasms. Invest New Drugs. 2010; 28 Suppl 1: S50-7.

8. Murawski N, Pfreundschuh M. New drugs for aggressive B-cell and T-cell lymphomas. Lancet Oncol. 2010; 11: 1074-85.

9. Giannini G, Cabri W, Fattorusso C, et al. Histone deacetylase inhibitors in the treatment of cancer: overview and perspectives. Future Med Chem. 2012; 4: 1439-60.

10. Khan O, La Thangue NB. HDAC inhibitors in cancer biology: emerging mechanisms and clinical applications. Immunol Cell Biol. 2012; 90: 85-94.

11. Jazirehi AR. Regulation of apoptosis-associated genes by histone deacetylase inhibitors: implications in cancer therapy. Anticancer Drugs. 2010; 21: 805-13.

12. Farnebo M, Bykov VJ, Wiman KG. The p53 tumor suppressor: a master regulator of diverse cellular processes and therapeutic target in cancer. Biochem Biophys Res Commun. 2010; 396: 85-9.

13. Sarita Rajender P, Ramasree D, Bhargavi K, et al. Selective inhibition of proteins regulating $\mathrm{CDK} /$ cyclin complexes: strategy against cancer-a review. J Recept Signal Transduct Res. 2010; 30: 206-13.

14. Swerdlow SH, Campo E, Harris NL, et al. WHO classification of tumours of haematopoietic and lymphoid tissues. Lyon: IARC press, 2008; 158-66.

15. Horwitz SM. The Emerging Role of Histone Deacetylase Inhibitors in Treating T-cell Lymphomas. Curr Hematol Malig Rep. 2011; 6: 67-72.
16. Min SK, Koh YH, Park Y, et al. Expression of HAT1 and HDAC1, 2, 3 in Diffuse Large B-Cell Lymphomas, Peripheral T-Cell Lymphomas, and NK/T-Cell Lymphomas. Korean J Pathol. $2012 ;$ 46:142-50.

17. Marquard L, Poulsen CB, Gjerdrum LM, et al. Histone deacetylase 1, 2, 6 and acetylated histone H4 in B- and T-cell lymphomas. Histopathology. 2009; 54: 688-98.

18. Adams H, Fritzsche FR, Dirnhofer S, et al. Class I histone deacetylases 1, 2 and 3 are highly expressed in classical Hodgkin's lymphoma. Expert Opin Ther Targets. 2010; 14: 577-84.

19. Marquard L, Gjerdrum LM, Christensen IJ, et al. Prognostic significance of the therapeutic targets histone deacetylase 1, 2, 6 and acetylated histone H4 in cutaneous T-cell lymphoma. Histopathology. 2008; 53: 267-77.

20. Müller BM, Jana L, Kasajima A, et al. Differential expression of histone deacetylases HDAC1, 2 and 3 in human breast cancer--overexpression of HDAC2 and HDAC3 is associated with clinicopathological indicators of disease progression. BMC Cancer. 2013; 13: 215.

21. Noh JH, Chang YG, Kim MG, et al. MiR-145 functions as a tumor suppressor by directly targeting histone deacetylase 2 in liver cancer. Cancer Lett. 2013; $335 ; 455-62$.

22. Du X, Zhao H, Zang L, et al. Overexpression of histone deacetylase 2 predicts unfavorable prognosis in human gallbladder carcinoma. Pathol Oncol Res. 2013; 19:397-403.

23. Tula-Sanchez AA, Havas AP, Alonge PJ, et al. A model of sensitivity and resistance to histone deacetylase inhibitors in diffuse large B cell lymphoma: Role of cyclin-dependent kinase inhibitors. Cancer Biol Ther. 2013; 14: 949-61.

24. Cycon KA, Mulvaney K, Rimsza LM, et al. Histone deacetylase inhibitors activate CIITA and MHC class II antigen expression in diffuse large B-cell lymphoma. Immunology. 2013; 140: 259-72. 